\title{
Portfolio Selection with Subsistence Consumption Constraints and CARA Utility
}

\author{
Gyoocheol Shim ${ }^{1}$ and Yong Hyun Shin ${ }^{2}$ \\ ${ }^{1}$ Department of Financial Engineering, Ajou University, Suwon 443-749, Republic of Korea \\ ${ }^{2}$ Department of Mathematics, Sookmyung Women's University, Seoul 140-742, Republic of Korea \\ Correspondence should be addressed to Yong Hyun Shin; yhshin@sookmyung.ac.kr
}

Received 2 January 2014; Revised 25 March 2014; Accepted 6 April 2014; Published 16 April 2014

Academic Editor: Pankaj Gupta

Copyright (c) 2014 G. Shim and Y. H. Shin. This is an open access article distributed under the Creative Commons Attribution License, which permits unrestricted use, distribution, and reproduction in any medium, provided the original work is properly cited.

\begin{abstract}
We consider the optimal consumption and portfolio choice problem with constant absolute risk aversion (CARA) utility and a subsistence consumption constraint. A subsistence consumption constraint means there exists a positive constant minimum level for the agent's optimal consumption. We use the dynamic programming approach to solve the optimization problem and also give the verification theorem. We illustrate the effects of the subsistence consumption constraint on the optimal consumption and portfolio choice rules by the numerical results.
\end{abstract}

\section{Introduction}

Following the seminal research works of Merton $[1,2]$, various problems of continuous-time optimal consumption and portfolio selection have been considered under various financial/economic constraints. One of the interesting research topics in a continuous-time portfolio selection problem is the optimization problem subject to a subsistence consumption constraint (or a downside consumption constraint). A subsistence constraint means that there exists a positive lower bound level for the agent's optimal consumption rate. Thus this constraint affects the agent's financial decision including her optimal portfolio.

Lakner and Nygren [3] have studied the portfolio optimization problem subject to a downside constraint for consumption and an insurance constraint for terminal wealth with a martingale approach. Gong and Li [4] have investigated the role of index bonds in the optimal consumption and portfolio selection problem with constant relative risk aversion (CRRA) utility and a real subsistence consumption constraint using the dynamic programming approach. Shin et al. [5] have also considered a similar problem to that of Gong and Li [4]. They have studied the portfolio selection problem with a general utility function and a downside consumption constraint using the martingale approach. Yuan and $\mathrm{Hu}$ [6] have investigated the optimal consumption and portfolio selection problem with a consumption habit constraint and a terminal wealth downside constraint using the martingale approach. In this paper we use the dynamic programming method based on Karatzas et al. [7] to derive the value function and the optimal policies in closed-form with a constant absolute risk aversion (CARA) utility function and a subsistence consumption constraint. Lim et al. [8] have considered a similar portfolio optimization problem combined with the voluntary retirement choice problem. Shin and Lim [9] have analyzed the effects of the subsistence consumption constraint for behavior of investment in the risky asset.

The rest of this paper proceeds as follows. Section 2 introduces the financial market. In Section 3 we consider the main optimization problem. We use the dynamic programming principle to derive the solutions in closed form with CARA utility and a subsistence consumption constraint. We also give some numerical results and the solutions derived by the martingale method. Section 4 concludes.

\section{The Financial Market Setup}

We assume that there are two assets in the financial market: one is a riskless asset with constant interest rate $r>0$, and the 
other is a stock whose price process $\left\{S_{t}\right\}_{t \geq 0}$ evolves according to the stochastic differential equation (SDE)

$$
\frac{d S_{t}}{S_{t}}=\mu d t+\sigma d B_{t}, \quad \text { for } t \geq 0,
$$

where $\mu$ is the constant expected rate of return of the stock, $\sigma>0$ is the constant volatility of the stock, and $B_{t}$ is a standard Brownian motion on a probability space $(\Omega, \mathscr{F}, \mathbb{P})$ endowed with the filtration $\left\{\mathscr{F}_{t}\right\}_{t \geq 0}$ which is the augmentation under $\mathbb{P}$ of the natural filtration generated by the standard Brownian motion $\left\{B_{t}\right\}_{t \geq 0}$. We assume that $\mu \neq r$ so that the market price of risk $\theta$ is not zero:

$$
\theta \triangleq \frac{\mu-r}{\sigma} \neq 0
$$

Let $X_{t}$ be an economic agent's wealth at time $t, \pi_{t}$ the amount of money invested in the stock at time $t$, and $c_{t}$ the consumption rate at time $t$. The portfolio process $\left\{\pi_{t}\right\}_{t \geq 0}$ is adapted to $\left\{\mathscr{F}_{t}\right\}_{t \geq 0}$ and satisfies, for all $t \geq 0$, almost surely (a.s.),

$$
\int_{0}^{t} \pi_{s}^{2} d s<\infty
$$

and the consumption rate process $\left\{c_{t}\right\}_{t \geq 0}$ is a nonnegative process adapted to $\left\{\mathscr{F}_{t}\right\}_{t \geq 0}$ such that, for all $t \geq 0$, a.s.,

$$
\int_{0}^{t} c_{s} d s<\infty
$$

We assume that there is a subsistence consumption constraint which restricts the minimum consumption level. That is, the consumption process should satisfy

$$
c_{t} \geq R, \quad \forall t \geq 0,
$$

where $R>0$ is a constant lower bound for the consumption rates. Thus the agent's wealth process $\left\{X_{t}\right\}_{t \geq 0}$ follows the SDE

$$
d X_{t}=\left[r X_{t}+\pi_{t}(\mu-r)-c_{t}\right] d t+\sigma \pi_{t} d B_{t},
$$

with an initial endowment $X_{0}=x>R / r$. (We need this restriction on the initial endowment for the positive consumption rate. See Lemma 3.1 of Gong and Li [4]). A consumption-portfolio plan $(\mathbf{c}, \pi):=\left(\left\{c_{t}\right\}_{t \geq 0},\left\{\pi_{t}\right\}_{t \geq 0}\right)$ satisfying the above conditions is called admissible at $x>R / r$ if $X_{t} \geq R / r$, for all $t \geq 0$. We let $\mathscr{A}(x)$ denote the class of admissible controls at $x>R / r$.

\section{The Optimization Problem}

Now the agent's optimization problem with initial wealth $X_{0}=x>R / r$ is to choose $(\mathbf{c}, \pi) \in \mathscr{A}(x)$ to maximize the following expected life-time utility:

$$
\mathbb{E}\left[\int_{0}^{\infty} e^{-\beta t} u\left(c_{t}\right) d t\right] .
$$

Here, $\beta>0$ is the subjective discount factor and $u(\cdot)$ is a constant absolute risk aversion (CARA) utility function defined by

$$
u(c) \triangleq-\frac{e^{-\gamma c}}{\gamma},
$$

where $\gamma>0$ is the agent's coefficient of absolute risk aversion. Thus the agent's value function is given by

$$
V^{*}(x) \triangleq \sup _{(\mathbf{c}, \pi) \in \mathscr{A}(x)} \mathbb{E}\left[-\int_{0}^{\infty} \frac{e^{-\beta t-\gamma c_{t}}}{\gamma} d t\right] .
$$

Bellman equation corresponding to the optimization problem for $x>R / r$ is

$$
\begin{gathered}
\max _{c \geq R, \pi}\left[\{r x+\pi(\mu-r)-c\} V^{\prime}(x)+\frac{1}{2} \sigma^{2} \pi^{2} V^{\prime \prime}(x)\right. \\
\left.-\beta V(x)-\frac{e^{-\gamma c}}{\gamma}\right]=0 .
\end{gathered}
$$

We assume that the wealth process $X_{t}$ must satisfy a transversality condition

$$
\lim _{t \rightarrow \infty} e^{-\beta t} V\left(X_{t}\right)=0 .
$$

We will find the solution $V(x)$, as the candidate value function, to Bellman equation (10) under the conditions that $V^{\prime}(x)>0$ and $V^{\prime \prime}(x)<0$ for $x>R / r$ and $V^{\prime}(\bar{x})=u^{\prime}(R)=$ $e^{-\gamma R}$ for a real number $\bar{x}>R / r$. After obtaining the solution, we can check these conditions. Under these conditions, in particular, the first-order condition (FOC),$-V^{\prime}(x)+u^{\prime}(c)=0$ with respect to $c \geq R$, is binding if $R / r<x<\bar{x}$ so that the maximizing $c \geq R$ in Bellman equation (10) is $R$ in this case.

Thus, from the first-order conditions (FOCs) of Bellman equation (10), we derive the candidate optimal consumption and portfolio

$$
\begin{aligned}
& c^{*}= \begin{cases}R, & \text { if } \frac{R}{r}<x<\bar{x} \\
-\frac{\log \left\{V^{\prime}(x)\right\}}{\gamma}, & \text { if } x \geq \bar{x},\end{cases} \\
& \pi^{*}=-\frac{\theta V^{\prime}(x)}{\sigma V^{\prime \prime}(x)} .
\end{aligned}
$$

Remark 1. For later use, we consider two quadratic algebraic equations:

$$
r m^{2}-\left(r+\beta+\frac{\theta^{2}}{2}\right) m+\beta=0,
$$

with two roots $m_{1}\left(0<m_{1}<1\right)$ and $m_{2}>1$ and

$$
\frac{\theta^{2}}{2 \gamma} n^{2}+\left(r-\beta-\frac{\theta^{2}}{2}\right) n-r \gamma=0,
$$

with two roots $n_{1}<0$ and $n_{2}>\gamma$. 
Theorem 2. Let $V(x)$ be given by

$$
V(x)=\left\{\begin{array}{cl}
C_{1}\left(x-\frac{R}{r}\right)^{m_{1}}-\frac{e^{-\gamma R}}{\beta \gamma}, & \text { if } \frac{R}{r}<x<\bar{x} \\
\frac{1}{\beta}\left(r+\frac{\theta^{2}}{2 \gamma} n_{1}\right) D_{1} e^{\left(n_{1}-\gamma\right) \xi} & \\
-\frac{1}{r \gamma} e^{-\gamma \xi}, & \text { if } x \geq \bar{x},
\end{array}\right.
$$

where

$$
\begin{gathered}
D_{1}=-\frac{r m_{1}-\beta-\theta^{2} / 2}{r^{2} \gamma e^{n_{1} R}\left(1-\left(\left(1-m_{1}\right) / \gamma\right) n_{1}\right)}>0, \\
\bar{x}=\frac{1-m_{1}}{\gamma}\left(n_{1} D_{1} e^{n_{1} R}+\frac{1}{r}\right)+\frac{R}{r}>\frac{R}{r}, \\
C_{1}=\frac{e^{-\gamma R}}{m_{1}}\left(\bar{x}-\frac{R}{r}\right)^{1-m_{1}}>0,
\end{gathered}
$$

and $\xi$ is determined from the algebraic equation

$$
x=D_{1} e^{n_{1} \xi}+\frac{1}{r} \xi+\frac{1}{r^{2} \gamma}\left(r-\beta-\frac{\theta^{2}}{2}\right) .
$$

Then it satisfies Bellman equation (10).

Proof. By using Remark 1, we can check the inequalities in (16) and (17). The inequality in (18) holds by (17). Define the function $X(c)$ of $c$ on $[R, \infty)$ by

$$
X(c)=D_{1} e^{n_{1} c}+\frac{1}{r} c+\frac{1}{r^{2} \gamma}\left(r-\beta-\frac{\theta^{2}}{2}\right) .
$$

By using (16) and (17), one can check

$$
X(R)=\bar{x} .
$$

Since the function $X(c)$ is increasing in $c$, it has the inverse function. Let $C(x)$ for $x \geq \bar{x}$ be the inverse function of $X(c)$. In particular, we have

$$
C(\bar{x})=R \text {. }
$$

By (19), we have

$$
\xi=C(x) \quad \text { for } x \geq \bar{x} .
$$

By using Remark 1, (16), (18), (22), and (23), we can show that the function $V(x)$ defined by (15) is continuous. By using Remark 1, (20), (23), and the inverse relationship between $X$ and $C$, we can obtain

$$
V^{\prime}(x)=e^{-\gamma C(x)}, \quad V^{\prime \prime}(x)=-\frac{\gamma e^{-\gamma C(x)}}{X^{\prime}(C(x))}, \quad \text { for } x>\bar{x}
$$

By (15), (18), (20), (22), and (24), we get the smooth-pasting $\left(C^{1}\right)$ condition

$$
V^{\prime}(\bar{x}-)=m_{1} C_{1}\left(\bar{x}-\frac{R}{r}\right)^{m_{1}-1}=e^{-\gamma R}=V^{\prime}(\bar{x}+),
$$

and the high-contact $\left(C^{2}\right)$ condition

$$
\begin{aligned}
V^{\prime \prime}(\bar{x}-) & =m_{1}\left(m_{1}-1\right) C_{1}\left(\bar{x}-\frac{R}{r}\right)^{m_{1}-2} \\
& =-\frac{\gamma e^{-\gamma R}}{X^{\prime}(R)}=V^{\prime \prime}(\bar{x}+) .
\end{aligned}
$$

Thus, the function $V(x)$ is twice continuously differentiable. Furthermore, $V^{\prime}(x)>0$ and $V^{\prime \prime}(x)<0$ for $x>R / r$ and $V^{\prime}(\bar{x})=u^{\prime}(R)=e^{-\gamma R}$.

For $R / r<x<\bar{x}$, if we substitute FOCs (12) into Bellman equation (10), we obtain the changed Bellman equation

$$
(r x-R) V^{\prime}(x)-\frac{1}{2} \theta^{2} \frac{\left(V^{\prime}(x)\right)^{2}}{V^{\prime \prime}(x)}-\beta V(x)-\frac{e^{-\gamma R}}{\gamma}=0 .
$$

We can easily check that $V(x)$ is the solution to (27) for $R / r<$ $x<\bar{x}$.

For $x \geq \bar{x}$, we also obtain the changed Bellman equation from (10):

$$
\begin{aligned}
r x V^{\prime}(x)-\frac{1}{2} & \theta^{2} \\
& \frac{\left(V^{\prime}(x)\right)^{2}}{V^{\prime \prime}(x)}-\beta V(x) \\
& +\frac{V^{\prime}(x)}{\gamma}\left(\log V^{\prime}(x)-1\right)=0 .
\end{aligned}
$$

If we substitute (24) into Bellman equation (28), then we obtain the equation

$$
\begin{gathered}
r X(c) e^{-\gamma c}+\frac{\theta^{2}}{2 \gamma} X^{\prime}(c) e^{-\gamma c}-\beta V(X(c)) \\
-\frac{e^{-\gamma c}}{\gamma}(\gamma c+1)=0 .
\end{gathered}
$$

By using (15) and (23), we can check that (29) holds.

Now we can derive the candidate optimal policies with the function $V(\cdot)$ in Theorem 2.

Theorem 3. The candidate optimal policies are given by $\left(c^{*}, \pi^{*}\right)$ such that

$$
c_{t}^{*}= \begin{cases}R, & \text { if } \frac{R}{r}<X_{t}<\bar{x} \\ \xi_{t}, & \text { if } X_{t} \geq \bar{x}\end{cases}
$$

$$
\pi_{t}^{*}= \begin{cases}\frac{\theta}{\sigma\left(1-m_{1}\right)}\left(X_{t}-\frac{R}{r}\right), & \text { if } \frac{R}{r}<X_{t}<\bar{x}, \\ \frac{\theta}{\sigma \gamma}\left(n_{1} D_{1} e^{n_{1} \xi_{t}}+\frac{1}{r}\right), & \text { if } X_{t} \geq \bar{x},\end{cases}
$$

where $\xi_{t}$ is determined from the optimal wealth process

$$
X_{t}=D_{1} e^{n_{1} \xi_{t}}+\frac{1}{r} \xi_{t}+\frac{1}{r^{2} \gamma}\left(r-\beta-\frac{\theta^{2}}{2}\right) .
$$


Theorem 4 (verification theorem). The value function of the optimization problem (9) is equal to $V(x)$ in Theorem 2. That is, $V^{*}(x)=V(x)$. Consequently the candidate consumption and portfolio in Theorem 3 are the optimal policies of problem (9).

Proof. For arbitrary given consumption and portfolio plan $(\mathbf{c}, \pi) \in \mathscr{A}(x)$ and $T \in(0, \infty)$, we have

$$
\begin{aligned}
& \mathbb{E}\left[-\int_{0}^{T} \frac{e^{-\beta t-\gamma c_{t}}}{\gamma} d t\right] \\
& \leq \mathbb{E}\left[-\int_{0}^{T} e^{-\beta t}\left\{\left(r X_{t}+\pi_{t}(\mu-r)-c_{t}\right) V^{\prime}\left(X_{t}\right)\right.\right. \\
& \left.\left.\quad+\frac{1}{2} \sigma^{2} \pi_{t}^{2} V^{\prime \prime}\left(X_{t}\right)-\beta V\left(X_{t}\right)\right\} d t\right] \\
& =\mathbb{E}\left[-\int_{0}^{T} d\left(e^{-\beta t} V\left(X_{t}\right)\right)\right] \\
& +\mathbb{E}\left[\int_{0}^{T} e^{-\beta t} \sigma \pi_{t} V^{\prime}\left(X_{t}\right) d B_{t}\right] \\
& =V(x)-\mathbb{E}\left[e^{-\beta T} V\left(X_{T}\right)\right],
\end{aligned}
$$

where the inequality is obtained from Bellman equation (10), the first equality from applying Itô's formula to $e^{-\beta t} V\left(X_{t}\right)$, and the second equality from $\mathbb{E}\left[\int_{0}^{T} e^{-\beta t} \sigma \pi_{t} V^{\prime}\left(X_{t}\right) d B_{t}\right]=0$. Taking $T \uparrow \infty$ and using transversality condition (11), we have

$$
V(x) \geq \mathbb{E}\left[-\int_{0}^{\infty} \frac{e^{-\beta t-\gamma c_{t}}}{\gamma} d t\right]
$$

for arbitrary given consumption and portfolio plan $(\mathbf{c}, \boldsymbol{\pi}) \epsilon$ $\mathscr{A}(x)$; that is,

$$
V(x) \geq \sup _{(\mathbf{c}, \pi) \in \mathscr{A}(x)} \mathbb{E}\left[-\int_{0}^{\infty} \frac{e^{-\beta t-\gamma c_{t}}}{\gamma} d t\right]
$$

Now we consider the candidate optimal consumption and portfolio plan $\left(\mathbf{c}^{*}, \boldsymbol{\pi}^{*}\right) \in \mathscr{A}(x)$ in Theorem 3 . For $T \in(0, \infty)$, we have

$$
\begin{array}{r}
\mathbb{E}\left[-\int_{0}^{T} \frac{e^{-\beta t-\gamma c_{t}^{*}}}{\gamma} d t\right] \\
=\mathbb{E}\left[-\int_{0}^{T} e^{-\beta t}\left\{\left(r X_{t}+\pi_{t}^{*}(\mu-r)-c_{t}^{*}\right) V^{\prime}\left(X_{t}\right)\right.\right. \\
\left.\left.+\frac{1}{2} \sigma^{2}\left(\pi_{t}^{*}\right)^{2} V^{\prime \prime}\left(X_{t}\right)-\beta V\left(X_{t}\right)\right\} d t\right]
\end{array}
$$

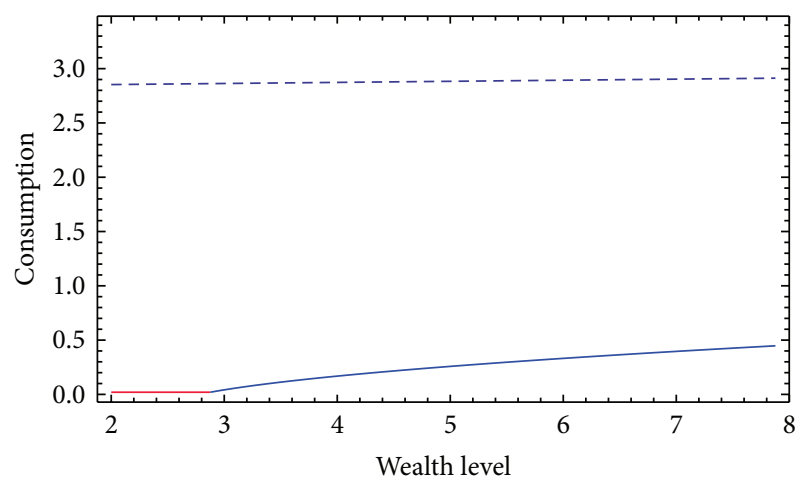

FIgURE 1: This figure is the optimal consumption rate $c_{t}^{*}$ when $\beta=$ $0.05, r=0.01, \mu=0.07, \sigma=0.2, R=0.02$, and $\gamma=3$. Solid line gives the optimal consumption rate with a subsistence consumption constraint and dotted line gives the optimal consumption rate without the constraint.

$$
\begin{aligned}
& =\mathbb{E}\left[-\int_{0}^{T} d\left(e^{-\beta t} V\left(X_{t}\right)\right)\right] \\
& \quad+\mathbb{E}\left[\int_{0}^{T} e^{-\beta t} \sigma \pi_{t}^{*} V^{\prime}\left(X_{t}\right) d B_{t}\right] \\
& =V(x)-\mathbb{E}\left[e^{-\beta T} V\left(X_{T}\right)\right],
\end{aligned}
$$

where the first equality is obtained from Bellman equation (10), the second from applying Itô's formula to $e^{-\beta t} V\left(X_{t}\right)$, and the third from $\mathbb{E}\left[\int_{0}^{T} e^{-\beta t} \sigma \pi_{t}^{*} V^{\prime}\left(X_{t}\right) d B_{t}\right]=0$. Taking $T \uparrow \infty$ and using transversality condition (11), we have

$$
V(x)=\mathbb{E}\left[-\int_{0}^{\infty} \frac{e^{-\beta t-\gamma c_{t}^{*}}}{\gamma} d t\right] .
$$

Thus, from (35) and (37), we show that $V(x)$ which is the solution to Bellman equation (10) is a real value function of the optimization problem (9).

Now we compare our solution in Theorem 3 with the Merton's solution with CARA utility. The optimal consumption and portfolio policies without the subsistence constraint are given by

$$
c_{t}^{M}=r X_{t}+\frac{1}{r \gamma}\left(\beta-r+\frac{1}{2} \theta^{2}\right), \quad \pi_{t}^{M}=\frac{\theta}{\sigma r \gamma},
$$

respectively. Figures 1 and 2 give the numerical results for the optimal consumption and portfolio.

Remark 5. Dynamic programming principle can be also applied to the CRRA utility function with a subsistence consumption constraint following our approach. See Gong and $\mathrm{Li}[4]$ and Lee and Shin [10].

Remark 6. Following Shin et al. [5] we can use the martingale method to derive a similar solution with CARA utility. We will give (rough) sketch of the derivation. 


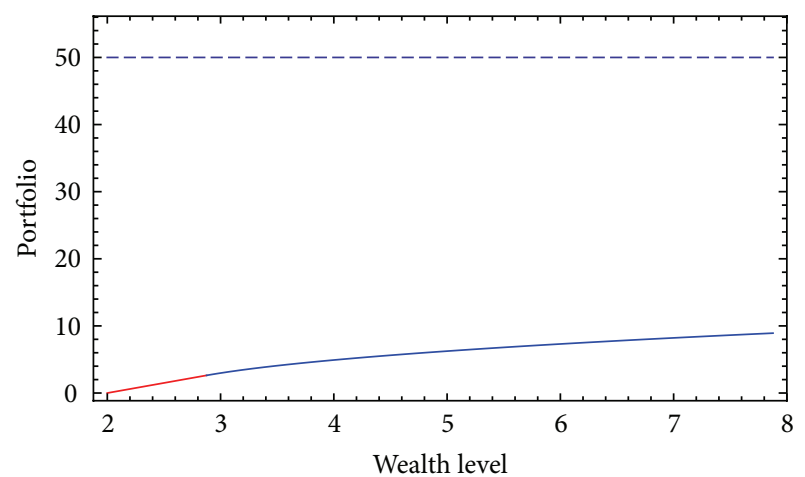

FIgURE 2: This figure is the optimal portfolio $\pi_{t}^{*}$ when $\beta=0.05, r=$ $0.01, \mu=0.07, \sigma=0.2, R=0.02$, and $\gamma=3$. Solid line gives the optimal portfolio with a subsistence consumption constraint and dotted line gives the optimal portfolio without the constraint.

From the agent's wealth dynamics $X_{t}$ in (6), we obtain the budget constraint

$$
\mathbb{E}\left[\int_{0}^{\infty} c_{t} H_{t} d t\right] \leq x
$$

where the state price density $H_{t}$ is defined by

$$
H_{t} \triangleq e^{-\left(r+(1 / 2) \theta^{2}\right) t-\theta B_{t}} .
$$

A dual utility function $\tilde{u}(y)$ of the CARA utility function $u(c)=-e^{-\gamma c} / \gamma$ is derived by

$$
\begin{aligned}
\tilde{u}(y)=\sup _{c \geq R}\left[-\frac{e^{-\gamma c}}{\gamma}-c y\right]= & {\left[\frac{1}{\gamma} y \log y-\frac{1}{\gamma} y\right] \mathbf{1}_{\{0<y \leq \bar{y}\}} } \\
& +\left[-\frac{e^{-\gamma R}}{\gamma}-R y\right] \mathbf{1}_{\{y>\bar{y}\}},
\end{aligned}
$$

where $\bar{y}=e^{-\gamma R}$. The dual value function $v(y)$ is given by

$$
\begin{aligned}
& v(y)=\mathbb{E}^{y_{0}=y}\left[\int _ { 0 } ^ { \infty } e ^ { - \beta t } \left[\left\{\frac{1}{\gamma} y_{t} \log \left(y_{t}\right)-\frac{1}{\gamma} y_{t}\right\} \mathbf{1}_{\left\{0<y_{t} \leq \bar{y}\right\}}\right.\right. \\
& \left.\left.+\left\{-\frac{e^{-\gamma R}}{\gamma}-R y_{t}\right\} \mathbf{1}_{\left\{y_{t}>\bar{y}\right\}}\right] d t\right] \\
& =\left\{\begin{aligned}
c_{1} y^{p_{+}}+\frac{1}{r \gamma} y \log y & \\
+\frac{1}{r^{2} \gamma}\left(\beta-2 r+\frac{1}{2} \theta^{2}\right) y, & \text { if } 0<y \leq \bar{y}, \\
d_{2} y^{p_{-}}-\frac{R}{r} y-\frac{e^{-\gamma R}}{\beta \gamma}, & \text { if } y>\bar{y},
\end{aligned}\right.
\end{aligned}
$$

where $y_{t} \triangleq y e^{\beta t} H_{t}$ and $p_{+}>1$ and $p_{-}<0$ are two real roots of the quadratic algebraic equation

$$
\frac{1}{2} \theta^{2} p^{2}+\left(\beta-r-\frac{1}{2} \theta^{2}\right) p-\beta=0 \text {. }
$$

The undetermined coefficients of $v(y)$ are given by

$$
\begin{aligned}
c_{1}= & \frac{\left(\left(p_{-}-1\right) / r^{2}\right)\left(\beta-2 r+(1 / 2) \theta^{2}\right)+p_{-} / \beta-1 / r}{\gamma\left(p_{+}-p_{-}\right)} \\
& \times e^{-\gamma R\left(1-p_{+}\right)}, \\
d_{2}= & \frac{\left(\left(p_{+}-1\right) / r^{2}\right)\left(\beta-2 r+(1 / 2) \theta^{2}\right)+p_{+} / \beta-1 / r}{\gamma\left(p_{+}-p_{-}\right)} \\
& \times e^{-\gamma R\left(1-p_{-}\right)} .
\end{aligned}
$$

Then we use the Legendre transform inverse formula to obtain the value function $V_{m}(x)$ as follows:

$V_{m}(x)$

$$
\begin{aligned}
& =\inf _{y>0}[v(y)+x y] \\
& =\left\{\begin{array}{cl}
\frac{p_{-}-1}{p_{-}}\left(-\frac{1}{p_{-} d_{2}}\right)^{1 /\left(p_{-}-1\right)} \\
\quad \times\left(x-\frac{R}{r}\right)^{p_{-} /\left(p_{-}-1\right)}-\frac{e^{-\gamma R}}{\beta \gamma}, & \text { if } \frac{R}{r}<x<\bar{x}_{m}, \\
\left(1-p_{+}\right) c_{1}\left(y^{*}\right)^{p_{+}}-\frac{1}{r \gamma}\left(y^{*}\right), & \text { if } x \geq \bar{x}_{m},
\end{array}\right.
\end{aligned}
$$

where

$$
\bar{x}_{m}=-p_{+} c_{1} e^{-\gamma R\left(p_{+}-1\right)}+\frac{R}{r}+\frac{1}{r^{2} \gamma}\left(r-\beta-\frac{1}{2} \theta^{2}\right),
$$

and $y^{*}$ is determined from the following algebraic equation:

$$
x=-p_{+} c_{1}\left(y^{*}\right)^{p_{+}-1}-\frac{1}{r \gamma} \log \left(y^{*}\right)+\frac{1}{r^{2} \gamma}\left(r-\beta-\frac{1}{2} \theta^{2}\right) .
$$

Actually we can show that $V_{m}(x)$ in (45) and $\bar{x}_{m}$ in (46) agree with $V(x)$ in (15) and $\bar{x}$ in (17), respectively, if we set $y^{*}=e^{-\gamma \xi}$ and show that

$$
m_{1} C_{1}=\left(-p_{-} d_{2}\right)^{1 /\left(1-p_{-}\right)}, \quad D_{1}=-p_{+} c_{1} .
$$

Refer to Lee and Shin [10].

Remark 7. We simplify the calculation by using the dynamic programming approach, where, instead of Legendre transformation in the martingale approach, we introduce the function $X(c)$ in (20) whose inverse is $C(x)$ in (23). The link between the two methods is the relation $y^{*}=e^{-\gamma \xi}=e^{-\gamma C(x)}=$ $u^{\prime}(C(x))=V^{\prime}(x)$ for $x \geq \bar{x}$, where the last equality is in (24).

\section{Concluding Remarks}

We have considered the optimal consumption and portfolio choice problem with constant absolute risk aversion (CARA) utility and a subsistence consumption constraint. The existence of a subsistence consumption constraint is 
realistic since one needs a minimum level of consumption to live. For example, we cannot live without necessities. We have obtained the closed form solution to optimization problem by using the dynamic programming approach. We have illustrated the effects of the subsistence consumption constraint on the optimal consumption and portfolio by the numerical results. Furthermore one can consider the optimization problem under regime switching as future research.

\section{Conflict of Interests}

The authors declare that there is no conflict of interests regarding the publication of this paper.

\section{Acknowledgments}

The authors are indebted to two anonymous referees for their helpful comments and insightful suggestions. The research of the corresponding author (Yong Hyun Shin) was supported by Sookmyung Women's University Research Grants 2012.

\section{References}

[1] R. C. Merton, "Lifetime portfolio selection under uncertainty: the continuous-time case," Review of Economics and Statistics, vol. 51, no. 3, pp. 247-257, 1969.

[2] R. C. Merton, "Optimum consumption and portfolio rules in a continuous-time model," Journal of Economic Theory, vol. 3, no. 4, pp. 373-413, 1971.

[3] P. Lakner and L. M. Nygren, "Portfolio optimization with downside constraints," Mathematical Finance, vol. 16, no. 2, pp. 283-299, 2006.

[4] N. Gong and T. Li, "Role of index bonds in an optimal dynamic asset allocation model with real subsistence consumption," Applied Mathematics and Computation, vol. 174, no. 1, pp. 710731, 2006.

[5] Y. H. Shin, B. H. Lim, and U. J. Choi, "Optimal consumption and portfolio selection problem with downside consumption constraints," Applied Mathematics and Computation, vol. 188, no. 2, pp. 1801-1811, 2007.

[6] H. Yuan and Y. Hu, "Optimal consumption and portfolio policies with the consumption habit constraints and the terminal wealth downside constraints," Insurance: Mathematics \& Economics, vol. 45, no. 3, pp. 405-409, 2009.

[7] I. Karatzas, J. P. Lehoczky, S. P. Sethi, and S. E. Shreve, "Explicit solution of a general consumption/investment problem," Mathematics of Operations Research, vol. 11, no. 2, pp. 261-294, 1986.

[8] B. H. Lim, Y. H. Shin, and U. J. Choi, "Optimal investment, consumption and retirement choice problem with disutility and subsistence consumption constraints," Journal of Mathematical Analysis and Applications, vol. 345, no. 1, pp. 109-122, 2008.

[9] Y. H. Shin and B. H. Lim, "Comparison of optimal portfolios with and without subsistence consumption constraints," Nonlinear Analysis: Theory, Methods \& Applications, vol. 74, no. 1, pp. 50-58, 2011.

[10] H. S. Lee and Y. H. Shin, "An optimal consumption and investment problem with subsistence consumption constraints: a dynamic programming approach," Working Paper, 2014. 


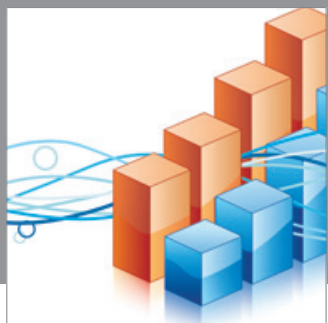

Advances in

Operations Research

mansans

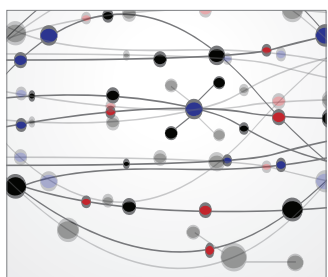

The Scientific World Journal
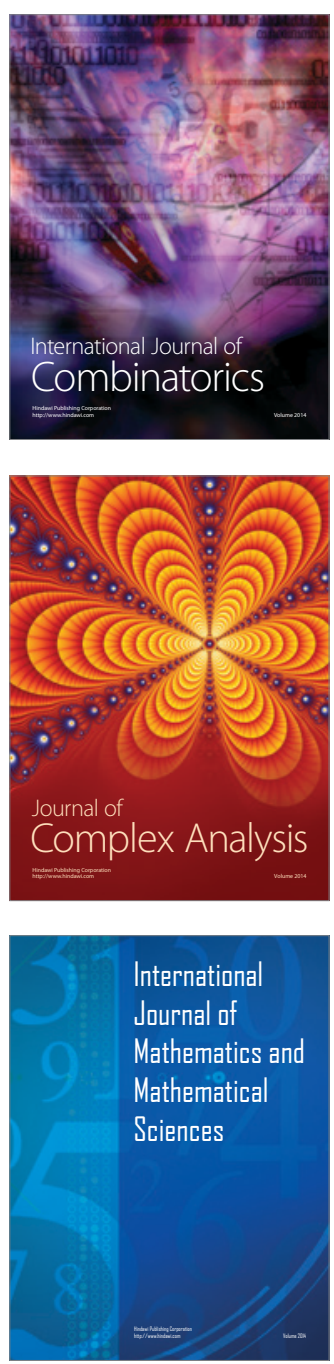
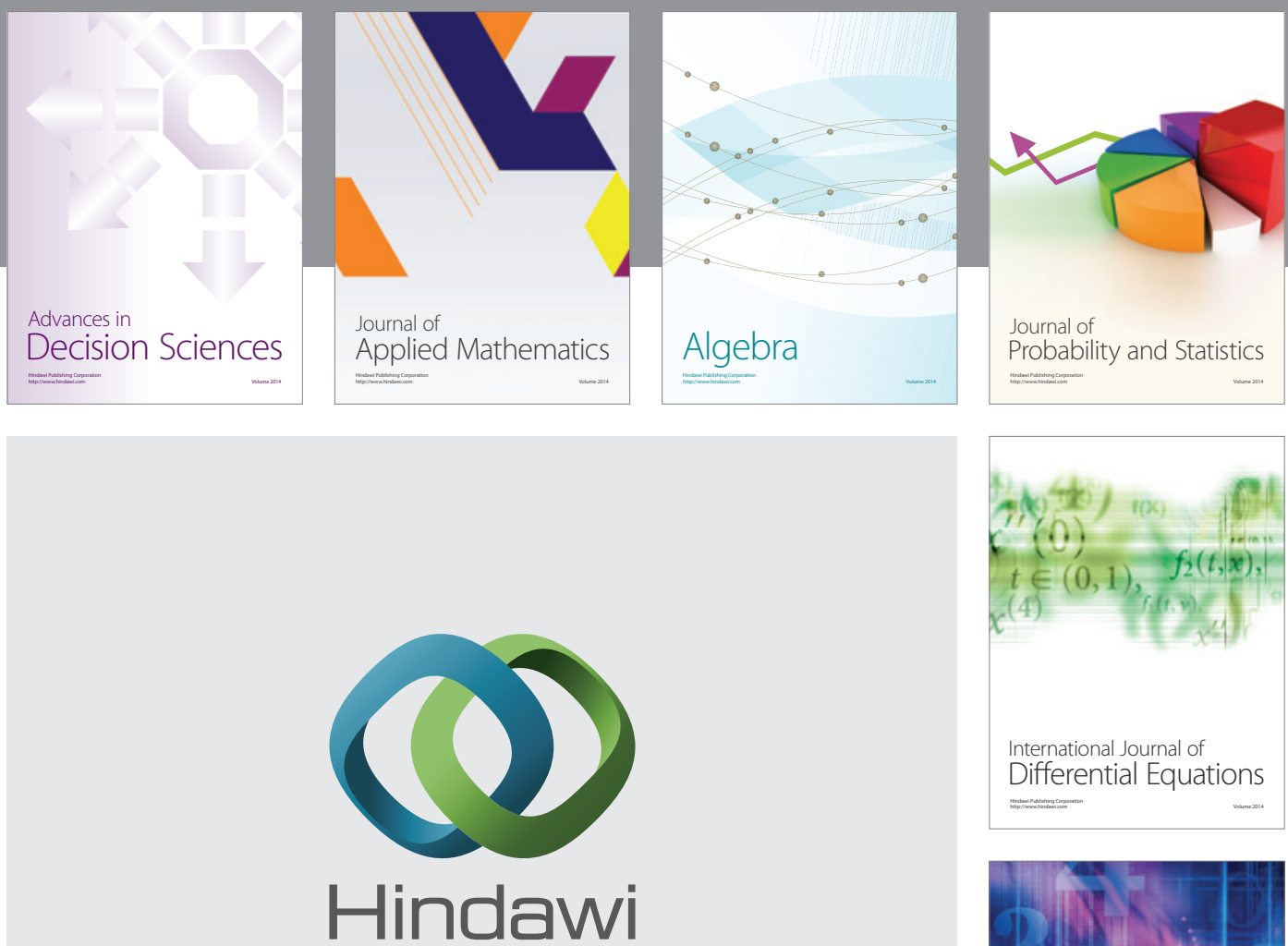

Submit your manuscripts at http://www.hindawi.com
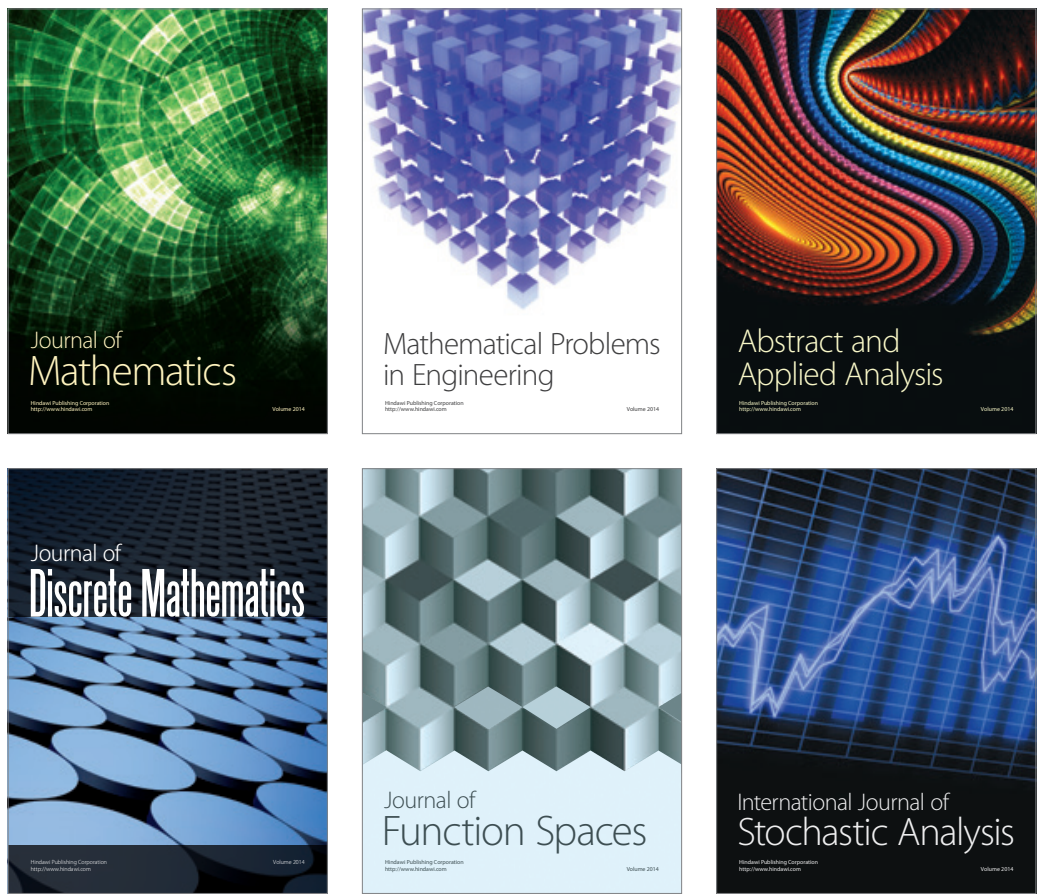

Journal of

Function Spaces

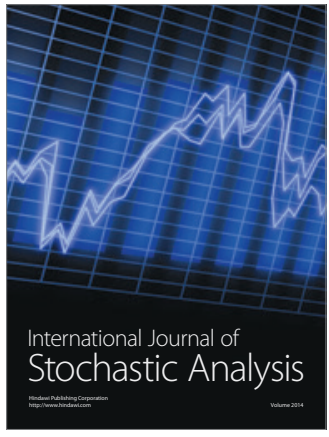

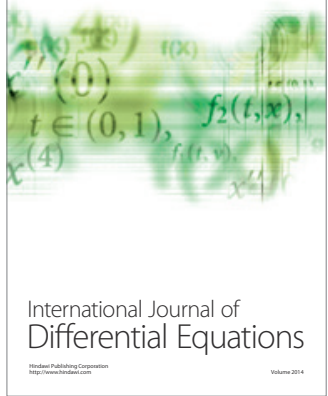
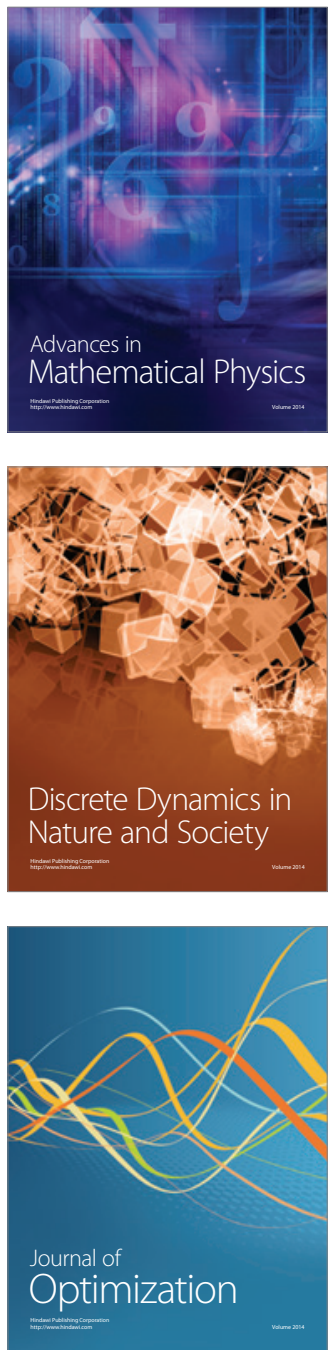